\title{
A novel model-based control strategy for aerobic filamentous fungal fed-batch fermentation processes
}

Mears, Lisa; Stocks, Stuart M.; Albaek, Mads O.; Cassells, Benny; Sin, Gürkan; Gernaey, Krist

Published in:

Biotechnology and Bioengineering (Print)

Link to article, DOI:

10.1002/bit.26274

Publication date:

2017

Document Version

Peer reviewed version

Link back to DTU Orbit

Citation (APA):

Mears, L., Stocks, S. M., Albaek, M. O., Cassells, B., Sin, G., \& Gernaey, K. (2017). A novel model-based control strategy for aerobic filamentous fungal fed-batch fermentation processes. Biotechnology and Bioengineering (Print), 114(7), 1459-1468 . https://doi.org/10.1002/bit.26274

\section{General rights}

Copyright and moral rights for the publications made accessible in the public portal are retained by the authors and/or other copyright owners and it is a condition of accessing publications that users recognise and abide by the legal requirements associated with these rights.

- Users may download and print one copy of any publication from the public portal for the purpose of private study or research.

- You may not further distribute the material or use it for any profit-making activity or commercial gain

- You may freely distribute the URL identifying the publication in the public portal 
A novel model-based control strategy for aerobic filamentous fungal fed-batch fermentation processes $^{\dagger}$

Lisa Mears $^{1}$, Stuart M. Stocks², Mads O. Albaek ${ }^{3}$, Benny Cassells ${ }^{3}$, Gürkan Sin $^{1}$, Krist V. Gernaey

${ }^{1}$ Department of Chemical and Biochemical Engineering, Technical University of Denmark, Kgs.

Lyngby, Denmark

${ }^{2}$ Leo Pharma A/S, Ballerup, Denmark

${ }^{3}$ Fermentation pilot plant, Novozymes A/S, Bagsværd, Denmark

Model-based fermentation control

Krist V. Gernaey

CAPEC-PROCESS Research Centre,

Department of Chemical and Biochemical Engineering

Technical University of Denmark

2800 Lyngby, Denmark

kvg@kt.dtu.dk

$+4545252970$

Fax: +45 45932906

${ }^{\dagger}$ This article has been accepted for publication and undergone full peer review but has not been through the copyediting, typesetting, pagination and proofreading process, which may lead to differences between this version and the Version of Record. Please cite this article as doi: [10.1002/bit.26274]

This article is protected by copyright. All rights reserved Received November 18, 2016; Accepted February 19, 2017 


\begin{abstract}
A novel model-based control strategy is developed for filamentous fungal fed-batch fermentation processes. The system of interest is a pilot scale (550L) filamentous fungus process operating at Novozymes A/S. In such processes, it is desirable to maximize the total product achieved in a batch in a defined process time. In order to achieve this goal, it is important to maximize both the product concentration, and also the total final mass in the fed-batch system. To this end, we describe the development of a control strategy which aims to achieve maximum tank fill, whilst avoiding oxygen limited conditions. This requires a two stage approach: (1) Calculation of the tank start fill and, (2) On-line control in order to maximize fill subject to oxygen transfer limitations. Firstly, a mechanistic model is applied off-line in order to determine the appropriate start fill for processes with four different sets of process operating conditions for the stirrer speed, headspace pressure and aeration rate. The start fills are tested with eight pilot scale experiments using a reference process operation. An on-line control strategy is then developed, utilising the mechanistic model which is recursively updated using on-line measurements. The model is applied in order to predict the current system states, including the biomass concentration, and to simulate the expected future trajectory of the system until a specified end time. In this way, the desired feed rate is updated along the progress of the batch taking into account the oxygen mass transfer conditions and the expected future trajectory of the mass. The final results show that the target fill is achieved to within 5\% under the maximum fill when tested using eight pilot scale batches, and over filling was avoided. The results were reproducible, unlike the reference experiments which show over $10 \%$ variation in the final tank fill, and this also includes over filling. The variance of the final tank fill is reduced by over $74 \%$, meaning that it is possible to target the final maximum fill reproducibly. The product concentration achieved at a given set of process conditions is unaffected by the control strategy. This article is protected by copyright. All rights reserved
\end{abstract}

Keyword's: Fermentation; Control; Modelling; Process optimization; Model-based control

\title{
Introduction
}

Industrial fermentation processes 
Fermentation processes are applied for production of a wide range of industrial products including alcohols, amino acids, vitamins and enzymes (Doran, 2013). A majority of industrial fermentation products are produced by fed-batch strategies (Bodizs et al., 2007; Woodley et al., 2013), where the aim is to regulate the rate of feed addition to the process in order to meet the metabolic demand for substrate without over feeding (Stocks, 2013). Overfeeding is detrimental to the process, as it may cause by-product formation (Henes and Sonnleitner, 2007; Villadsen et al., 2011), or in highly viscous processes may lead to oxygen limitation (Bodizs et al., 2007; Posch et al., 2013). It is therefore desirable to regulate the feed addition in coordination with the biomass concentration, the metabolic rates, and the oxygen availability; however this is challenging as these three variables are constantly changing over the process duration.

The method of feed rate regulation has traditionally been by applying a predetermined feed rate profile in an open loop operation, as many are still operated today (Oliveira et al., 2004). It is also possible to manipulate the feed rate in closed loop feed-back control using, for example, the dissolved oxygen as a control variable (Albaek et al., 2011; Bodizs et al., 2007; Lee et al., 1999). A wide range of more advanced control strategies are also developed in literature, although there is limited application to industrial processes.

\section{Control objective}

The control objective for the fermentation process is to maximize the total product produced per batch, given a scheduled batch time. In order to maximize the product mass, it is possible to either increase the product concentration or to increase the mass in the system. There are many control strategies which specifically aim to increase the product concentration obtained from a fed-batch fermentation process (Chang et al., 2016; Kovárová-Kovar et al., 2000; Peng et al., 2013). Increasing the product concentration is often focused on reducing by-product formation, which is a waste of substrate, and a burden for the process. However, in industrial fermentation processes, the 
host strains are highly engineered and optimized, and many of the pathways associated with byproduct formation are removed to optimize productivity (Cherry and Fidantsef, 2003; Lee and Kim, 2015). This is the situation with the industrial strain of interest in this work where we assume the host strain is optimized to maximize the product yield. For this reason, it is considered that the final mass in the system is the factor which can most greatly impact the total product mass in the production phase.

Maximizing the fill of a fed-batch process is not a trivial problem. The system must be initiated at a level which means the target is reachable, given the changing oxygen transfer conditions of the system. For example in the work of Albaek et al. (2012) the oxygen mass transfer coefficient is seen to reduce by over $75 \%$ over the process time (Albaek et al., 2012). The oxygen transfer rate in the system at a given time, limits the feed rate which may be applied, in order to maintain aerobic process conditions. This rate is constantly changing, as the biomass concentration increases and fungal morphology changes, and therefore the viscosity increases, meaning a reduction in the oxygen availability. This is especially relevant for filamentous fungus processes, which can form a highly viscous broth, meaning that the oxygen transfer rate reduces significantly over the process time. This means that if the start fill of the batch is too low, it will not be possible to achieve the target in a fixed batch time. This dependence on the rheological properties of the broth, as well as the specific substrate uptake rates, means that the optimal start fill is therefore strain specific. For a given strain, the start fill is also dependent on the process operating conditions for the batch. This is for example with respect to the headspace pressure, the agitation rate, aeration rate, and temperature all of which affect the oxygen transfer to the broth. This means that the start fill is not only strain specific, but also dependent on the process operating conditions.

The start fill is also dependent on the equipment used. Different tank dimensions, and different impeller types, number and configurations will result in a system with different oxygen transfer 
rates. It is therefore not correct to assume the optimal start fill is equivalent between equipment, without first an assessment of the physical conditions in each system. Similarly, it is not possible to apply the same relative start fill to two different scales of tank. This is related to the well documented issues of process scale up, as described in detail in Stocks (2013). If a start fill is defined for a pilot scale process, the same relative fill is not directly applicable to a larger scale process, as the physical conditions do not scale linearly with size. For example, the additional hydrostatic pressure in a large process increases the oxygen solubility, and improves oxygen transfer. The Reynolds number is also greater at scale, due to the proportionality to the square of the agitator diameter, resulting in turbulent mixing at lower stirrer speeds (Stocks, 2013). Overall, it is clear that the start fill is dependent on the strain, the process conditions, the scale of operation, and the equipment used.

Assuming an appropriate start fill is identified for a fermentation process, there are then many process disturbances which mean that the same final fill is not achieved for a fixed start fill. This is due to many batch-to-batch uncertainties which may be experienced:

- The batch phase of a fed-batch process may vary in length due to different initial biomass concentration, age of the inoculum culture, or other batch-to-batch variations. This means that the feeding time may not be equal in every batch, even if the full batch time is equal.

- The feed concentration is subject to batch-to-batch variation, meaning the total mass added for a given mass of substrate is not equal in every batch

- Physiological differences between batches, for example mean growth rates, yields and maintenance requirements are slightly different, resulting in different metabolic rates - Evaporation rates are dependent on the outside air conditions which are not constant Therefore, for a given process, it is expected that even with the same start fill, there will be a 
considerable variation in the final fill. This variation may be reduced by means of a control strategy which aims to target a maximum batch fill.

The concept of this work is to reduce the variance in the final batch fill and consistently achieve maximum tank capacity, i.e. increase reproducibility. Due to the batch-to-batch variations however, if we reduce the variance in one process parameter (tank fill) it must then allow for variation in another process parameter. By analysing the relationships between the process variables it is seen that this may be achieved by allowing increased variation in the dissolved oxygen concentration in the system. Although it is important to avoid absolute oxygen limitation, it is considered in this work, to allow for a variation in the dissolved oxygen level as a trade-off for achieving a maximum tank fill. An additional benefit of achieving a reproducible maximum tank fill is also that it has benefits for planning and scheduling of downstream operations, and resource allocation.

In this work a control strategy and two implementation configurations (direct model-based control and a cascade configuration with regulatory layer) are described for controlling a fed-batch process to achieve maximum fill capacity subject to the oxygen transfer limitations of the system. The strategy also includes a batch planning step where an appropriate start fill is calculated by use of a mechanistic process model. The strategy is comprehensively tested at industrially relevant pilot scale studies (16 experiments in total) by testing also at different process operating conditions for headspace pressure, aeration rate and agitator speed. We consider this a novel approach, as to the best of our knowledge there is no similar control algorithm described in the literature.

\section{Materials and Methods}

The following section describes the Materials and Methods for this work, which includes the fermentation process description, model description, and the control algorithm description.

\section{Fermentation process}


The fermentation process is a pilot scale (550 L) fed-batch operation using a proprietary filamentous fungus strain, operated at Novozymes A/S. The process begins with a batch phase, where the operating conditions for stirrer speed, headspace pressure and aeration rate are set to minimal values. Once the batch phase of substrate is consumed, feeding begins, and the operating conditions are ramped to their designated set points over a fixed time period. Four different sets of operating conditions are tested, as shown in Table 1 . Table 1 also shows the tank numbers representing the different operating conditions and the plot colors for future reference in the text. Four fermentation processes are run in parallel, where the four different operating conditions are applied. This is referred to as one experiment consisting of four tanks. Four experiments were conducted in this work; two utilising a DO controller as described by Albaek et al. (2011) as a reference, and two utilising the model-based controller described in this work. Other controlled variables include $\mathrm{pH}$ and temperature, which are controlled to fixed set points.

\section{Mechanistic process model}

A mechanistic process model has been developed and validated for on-line application, as described in Mears et al. (2016). In this work, measurement data is used for parameter estimation in order to update formation rates of biomass and product by a stoichiometric balance approach. The required measurements are carbon evolution rate (CER), oxygen uptake rate (OUR) and ammonia flow rate. For off-line batch planning however, measurement data is not available, and therefore it is not possible to solve the formation rates. In this case, the biomass growth rate term, maintenance terms, and product yield terms are used, as described in Albaek et al. (2011). Model parameters are also refitted using a least squares approach in order to fit the model states. The physical process model remains the same, it is the metabolic rates which are replaced for off-line applications.

\section{Model-based batch planning for initial conditions}


The mechanistic model is applied in order to optimize the initial conditions for processes operated at four different operating conditions for stirrer speed, headspace pressure, and aeration rate, as shown in Table 1. It is important to test the methods at different process operating conditions as this strategy must be relevant for industrial application, where different equipment at different locations may have different limitations for the operating conditions achievable. For this initial study it also allows for robustness testing as it challenges the physical process model. The dynamic model is implemented as a non-linear optimization problem, in order to calculate the feed rate required to achieve a given DO profile. Based on the different oxygen transfer conditions, the batches will have different capacities for feed. The non-linear model predicts the batch mass, due to feed addition and evaporation rate (Mears et al., 2016). It is therefore possible to obtain the desired starting fill for the batch, considering the maximum tank fill and the oxygen transfer conditions. The maximum tank fill is defined as $410 \mathrm{~kg}$, and the batch time is fixed for all tanks and all experiments. Maximum feed rate constraints are also applied.

\section{Mode- based control strategy}

The concept of the controller is to simulate the process model until the end of the batch time in order to identify if the current batch fill is expected to be above or below the fill target. The target fill is defined as $410 \mathrm{~kg}$, which allows for sufficient headspace in the vessel. Adjustments are then made to the feed rate to drive the process towards a desired tank fill in a set process time. When the regulatory layer is also added, the calculated feed rate is adjusted by a proportional controller, in order to avoid oxygen limitation. This regulatory layer is only applied in a specified dead band, as it is not necessary to strictly regulate the oxygen profile, but just to avoid over and under feeding. This may occur due to process model mismatch, but should be avoided in order to maintain a productive process.

The control strategy is best described in Figures 1 and 2. The controller is implemented in Matlab 
(The MathWorks Inc., 2013), and consists of timers which run scripts for the measurement data collection, supervisory layer model simulation, and the regulatory layer. In Experiment 3, only the supervisory layer is implemented and this feed is applied directly to the process. In Experiment 4, the supervisory layer and the regulatory layer is implemented, as shown in Figure 1. The algorithm for the controller implementation is described in detail in Figure 2. There is a Matlab connection to the OPC server in order to access on-line measurement data, and also to write set points which are then applied to the system via the distributed control system (DCS).

\section{Results and Discussion}

In the following results section, the experiments are described by an experiment number as follows:

1. DO controller trial (reference)

2. DO controller trial (reference)

3. Model-based control

4. Model-based control with regulatory layer

The first two DO controlled experiments were run in order to assess the suitability of the calculated start fill. This is important as a reference for the performance of the model controlled batches.

Experiment 3 was controlled using the model-based controller, where the supervisory layer feed rate was applied directly. Experiment 4 employed the model-based controller algorithm, with an additional regulatory layer, as described in the Materials and Methods section.

\section{Model-based batch planning}

In order to target the final batch fill it is necessary to identify also a suitable batch start fill, depending on the given process limitations in terms of operating conditions. Table 1 shows the 
results of the start fill calculation which is obtained by simulating the full process using the different operating conditions. It is seen that the difference in the stirrer speed has the greatest impact on the start fill, as it has the greatest impact on the oxygen transfer. Of course, without absolute values of the process parameters, this is of limited value to the reader, however it will be important to identify if this trend is also represented in the experimental data. For example, if the lowest start fill is also the lowest end fill, then it is likely that the start fill was not appropriately determined. The start fill ranges from $39 \%$ to $77 \%$ depending on the processing conditions. In order to validate if this difference is of correct magnitude, it is necessary to run experiments utilising the different start fills. It is not desirable to run the model-based controller for this purpose, as we wish to identify the variation in final fill for the given start fills. Therefore dissolved oxygen control is applied, as a reference controller, using the feed rate as manipulated variable using a PI controller.

The results for the DO controlled batches (experiments 1 and 2) are shown in Figure 3. The start fill and final fill is shown for the two experiments, each run with the four different operating conditions, as described in Table 1. It is seen that the fill of Experiment 2 is noticeably lower for all conditions than Experiment 1, despite the very similar start fills. This emphasizes the relevance of the evaporation rate, in the long running aerated processes (Mears et al., 2016).

The two experiments were operated three months apart, and it therefore would be expected that they are subject to different air humidity and temperatures. In order to assess the difference in the evaporation rates at the two experiments, it is possible to look at the batch phase of the experiment, and see the difference in mass change over this period of the operation. In this phase there is no mass addition, and all experiments have the same operating conditions; it is only after the feed start that the process conditions are adjusted for the different tank numbers. Figure 4 shows the batch phase mass change for the two experiments and for all batches. Experiment 2 has a greater mass change than Experiment 1. This explains the deviation in final mass between the experiments in 
Figure 3. It is important to ensure that the model is able to capture this difference. When the actual air temperature and humidity was used as input to the evaporation model, the rate of evaporation from Experiment 2 is around 10\% greater than Experiment 3 due to the air conditions, so the model is seen to reproduce the trend seen in the data.

Due to the high fill levels seen in Experiment 1, some manual interventions were made to ensure that the tanks would not in fact over fill, which is a serious problem in a production facility where biological material must be contained at all times. For this reason, tanks 2 and 3 had small reductions in their stirrer speeds in Experiment 1, since they reached the point where they were 10\% over the target fill. This was done as a safety precaution. This also highlights the need for control of the fill in a tank, as it can be hazardous to allow the tank to become over full.

Overall, the results from Experiment 1 and Experiment 2 show that the calculated start fills are of suitable magnitude given the different operating conditions. Focussing on Experiment 2, where no changes were made to the operating conditions specified, the difference in start fill of $156 \mathrm{~kg}$ was reduced to a difference of only $24 \mathrm{~kg}$ in the final fill. This large reduction in variance shows that there is indeed a robust process model which is applicable to defining an appropriate start fill given a set of operating conditions. However these two experiments have also emphasized that the evaporation rate is of significance and must be considered as a variable for the process operation, not as a constant. For this reason also, the start fill is not fixed at an optimum, but will be dependent on the relative humidity and temperature of the outside air.

A final observation from the DO controlled batches is in fact that the dissolved oxygen is not able to be controlled for the full process time in the experiments with poor oxygen transfer. It is seen in Figure 5, that the dissolved oxygen profiles for the two experiments and for all tanks are able to follow the set point trajectory for around three quarters of the operation time, however in the final 
stages the oxygen transfer in tanks 3 and 4 is insufficient to maintain the DO at the set point. In Experiment 1 the minimum feed rate was not zero but a minimum feed rate value, so in Experiment 2 the minimum feed rate was changed to zero to see if the dissolved oxygen could be controlled to set point, but the same trend is seen. This is a result of the extreme conditions which are being tested in experiments 3 and 4, as shown in Table 1, where the oxygen transfer is too low at the end of the batch where the viscosity of the fermentation broth is high and therefore the oxygen concentration falls rapidly. This is not desirable, however this is also the purpose of running the DO batches as a reference case, so that it is possible to identify limitations in the operation. It is not expected that any other control operation should be able to maintain aerobic conditions at this late stage in the process for tanks 3 and 4.

\section{Model-based control}

Now that the start fill calculation has been verified by experiments, and we also have a reference operation performance for the controller, it is possible to test the model-based control strategy. Two experiments were conducted using the model-based control strategy as described in the Materials and Methods section. The difference between Experiment 3 and 4 is the addition of a regulatory layer to the control strategy implemented in Experiment 4.

The results for the final batch fill are shown in Figure 6. Experiment 3, Tank 1 is not shown, as this batch was lost due to technical issues, not related to the controller implementation. It is seen that the variation in final fill is reduced to within $5 \%$ of the target, and only under filling occurs. In the DO controlled batches, both over and under filling of around $10 \%$ of the target is seen, and as previously described, the level of over filling would have been greater if there was not manual intervention. This shows that the combination of the calculated start fill and the model-based control is successful to reliably target a desired tank fill. There is a difference in the results for the model-based control (Experiment 3) compared to the model-based control with regulatory layer 
(Experiment 4). The target fill is achieved in the designated batch time with an accuracy of $2 \mathrm{~kg}$ in Experiment 3, whereas there is under filling of around 5\% in Experiment 4. This is due to the tradeoff between the target fill and the oxygen limitations, which the controller accounts for as the batch is progressing.

Figure 7 shows the dissolved oxygen profiles for the two different controller types in comparison to the profiles for the dissolved oxygen controlled batches. Beneath this are the corresponding feed rate profiles. Tank 2 is chosen as comparison as there was good performance of the DO controller. It is seen that the model derived feed rate which is applied directly to the system in Experiment 3, is too high for the system, resulting in dissolved oxygen limitations. The feed rate calculated is significantly higher than that applied when using the dissolved oxygen controller. As previously explained, the aim is not to achieve a certain oxygen profile, but to avoid oxygen limitation, and therefore the feed rate should be close to that of the DO controller. When the regulatory layer is incorporated into the controller algorithm, the feed rate is successfully reduced at the end of the batch in order to avoid oxygen limitation. It is possible to adjust the $\mathrm{DO}_{\max }$ and $\mathrm{DO}_{\min }$ ramps in order to adjust at which point the regulatory layer will be activated. It is also possible to tune the controller gain, in order to respond faster and more aggressively to a value outside of the $\mathrm{DO}_{\max }$ and $\mathrm{DO}_{\min }$ ramps. Based on the aims of this work however, it is considered that Experiment 4 successfully achieves both the desired target fill, and with an acceptable dissolved oxygen profile, and that the controller was tuned suitably. Both the mass target is met to within 5\%, and oxygen limitation is avoided.

Figure 8 shows the conclusion for this work, where the dissolved oxygen control is compared to the model-based controller with regulatory layer. It is seen that the mass target is reached with a low variance in Experiment 4 in comparison to the variance seen when using the dissolved oxygen controller. This means that the controller accurately targets the desired final mass target with high 
reproducibility with the four different operating conditions tested. This means that there is a more reliable and consistent fill in each batch. In addition, the variance seen in the dissolved oxygen concentration is lower than when using the dissolved oxygen controller, due to overfeeding issues as previously described. In fact, the concept of the controller was to shift the variance from fill to dissolved oxygen, but using the model-based strategy with regulatory layer it is possible to have a reproducible final mass, whilst maintaining a desirable profile for the dissolved oxygen concentration in the system.

The overall objective for the control strategy is to maximize the total product mass from the system, so it is important to ensure that the model-based control does not have a detrimental effect on the product concentration. Figure 9 shows that there is no significant affect, either positive or negative, on the product concentration between the control strategies applied in this work.

\section{Conclusions}

In this work a novel control strategy is developed in order to maximize the fill in fed-batch fermentation processes. The target fill was achieved accurately, and with high reproducibility when tested with different process operating conditions, and different meteorological conditions. This is achieved by targeting a desired tank fill in a specified time, whilst avoiding oxygen transfer limitations during progression of batch operation. This is a novel control concept.

The control strategy has been rigorously and successfully tested in pilot scale (550 L) studies by performing in total 16 runs, including the reference control strategy, under four different industrially relevant process operating conditions. The results for the model-based control strategy with regulatory layer has been shown to meet this objective, with the target mass reached to within $5 \%$ under the target, and hence avoiding over filling. The reduction in variance of the final fill of over $74 \%$ is not only beneficial for the final product mass, but also for planning and scheduling in a 
multi-product facility, where downstream operations must also be scheduled. With a reliable product mass flow to recovery, resource allocation is more predictable.

In conclusion, focussing on final batch fill is a highly valuable control objective, especially in industry where highly optimized strains are applied. In the fermentation control literature, there are often references to control methods to obtain high product concentration, however in highly engineered industrial hosts we feel there are greater benefits in optimising total mass, than optimising product mass fraction. In this way it is not only possible to achieve maximum product mass in a batch operation, but it allows for predictable product mass to the downstream operations, which allows for improved scheduling and capacity planning.

Future work for this project includes a consideration of gas hold up in the fermentation system, to determine the optimal maximum fill, which in this work has been fixed based on prior experience.

\section{Acknowledgements}

The PhD project of Lisa Mears is funded by Novozymes A/S and the Technical University of Denmark (DTU). The authors would like to thank the Novozymes fermentation pilot plant operators for running of the fermentation systems presented in this work. 


\section{Nomenclature}

\begin{tabular}{|c|c|c|}
\hline CER & Carbon dioxide evolution rate & $\mathrm{mol} / \mathrm{h}$ \\
\hline $\mathrm{DO}$ & Dissolved oxygen concentration & $\mathrm{mol} / \mathrm{kg}$ \\
\hline $\mathrm{DO}_{\text {profile }}$ & Dissolved oxygen profile & $\%$ \\
\hline $\mathrm{DO}_{\max }$ & Maximum DO profile & $\%$ \\
\hline $\mathrm{DO}_{\min }$ & Minimum DO profile & $\%$ \\
\hline $\mathrm{F}$ & Feed rate & $\mathrm{L} / \mathrm{h}$ \\
\hline $\mathrm{FO}_{\text {limit }}$ & Maximum F/OUR & $\mathrm{L} / \mathrm{mol}$ \\
\hline F profile & Feed rate model trajectory & $\mathrm{L} / \mathrm{h}$ \\
\hline$F_{\text {supervisory }}$ & Feed rate from supervisory layer & $\mathrm{L} / \mathrm{h}$ \\
\hline HSP & Headspace pressure & barg \\
\hline $\mathrm{K}_{\mathrm{c}}$ & Controller proportional gain & $(\mathrm{L} / \mathrm{h}) / \%$ \\
\hline M & Mass & $\mathrm{kg}$ \\
\hline Mend & End mass prediction from model trajectory & $\mathrm{kg}$ \\
\hline $\mathrm{M}_{\text {target }}$ & Target end mass & $\mathrm{kg}$ \\
\hline $\mathrm{M}_{\mathrm{t}=0}$ & Starting mass & $\mathrm{kg}$ \\
\hline $\mathrm{NH}_{3}$ & Ammonia addition & $\mathrm{mol} / \mathrm{h}$ \\
\hline $\mathrm{N}$ & Stirrer speed & $\mathrm{rpm}$ \\
\hline OUR & Oxygen uptake rate & $\mathrm{mol} / \mathrm{h}$ \\
\hline Q & Air flowrate & $\mathrm{NL} / \mathrm{min}$ \\
\hline $\mathrm{q}_{\mathrm{x}}$ & Biomass formation rate & $\mathrm{mol} / \mathrm{h}$ \\
\hline$S_{\text {feed }}$ & Concentration of feed & $\mathrm{g} / \mathrm{kg}$ \\
\hline tend & End time & $\mathrm{h}$ \\
\hline
\end{tabular}




\section{References}

Albaek MO, Gernaey K V, Hansen MS, Stocks SM. 2011. Modeling enzyme production with Aspergillus oryzae in pilot scale vessels with different agitation, aeration, and agitator types. Biotechnol. Bioeng. 108:1828-40.

Albaek MO, Gernaey K V, Hansen MS, Stocks SM. 2012. Evaluation of the energy efficiency of enzyme fermentation by mechanistic modeling. Biotechnol. Bioeng. 109:950-61.

Bodizs L, Titica M, Faria N, Srinivasan B, Dochain D, Bonvin D. 2007. Oxygen control for an industrial pilot-scale fed-batch filamentous fungal fermentation. J. Process Control 17:595606.

Chang L, Liu X, Henson MA. 2016. Nonlinear model predictive control of fed-batch fermentations using dynamic flux balance models. J. Process Control 42:137-149.

Cherry JR, Fidantsef AL. 2003. Directed evolution of industrial enzymes: an update. Curr. Opin. Biotechnol. 14:438-443.

Doran PM. 2013. Bioprocess Development: An Interdisciplinary Challenge. In: . Bioprocess Eng. Princ. 2nd ed. Academic Press, pp. 3-11.

Henes B, Sonnleitner B. 2007. Controlled fed-batch by tracking the maximal culture capacity. J. Biotechnol. 132:118-26.

Kovárová-Kovar K, Gehlen S, Kunze A, Keller T, von Däniken R, Kolb M, van Loon APGM. 2000. Application of model-predictive control based on artificial neural networks to optimize the fed-batch process for riboflavin production. J. Biotechnol. 79:39-52.

Lee J, Lee SY, Park S, Middelberg APJ. 1999. Control of fed-batch fermentations. Biotechnol. Adv. 17:29-48. 
Lee SY, Kim HU. 2015. Systems strategies for developing industrial microbial strains. Nat.

Biotechnol. 33:1061-1072.

Mears L, Stocks SM, Albaek MO, Sin G, Gernaey K V. 2016. Application of a Mechanistic Model as a Tool for On-line Monitoring of Pilot Scale Filamentous Fungal Fermentation ProcessesThe Importance of Evaporation Effects. Biotechnol. Bioeng. Accepted.

Oliveira R, Simutis R, Feyo de Azevedo S. 2004. Design of a stable adaptive controller for driving aerobic fermentation processes near maximum oxygen transfer capacity. J. Process Control 14:617-626.

Peng J, Meng F, Ai Y. 2013. Time-dependent fermentation control strategies for enhancing synthesis of marine bacteriocin 1701 using artificial neural network and genetic algorithm. Bioresour. Technol. 138:345-52.

Posch AE, Herwig C, Spadiut O. 2013. Science-based bioprocess design for filamentous fungi. Trends Biotechnol. 31:37-44.

Stocks SM. 2013. Industrial enzyme production for the food and beverage industries: process scale up and scale down. In: McNeil, B, Archer, D, Giavasis, I, Harvey, L, editors. Microb. Prod. Food Ingredients, Enzym. Nutraceuticals. Cambridge: Woodhead Publishing Limited, pp. 144-172.

The MathWorks Inc. 2013. Matlab 8.2 (R2013b).

Villadsen J, Nielsen J, Lidén G. 2011. Bioreaction Engineering Principles Third. New York: Springer.

Woodley JM, Breuer M, Mink D. 2013. A future perspective on the role of industrial biotechnology 
for chemicals production. Chem. Eng. Res. Des. 91:2029-2036.

\section{FIGURE CAPTION:}

Figure1: Diagram showing the control structure for the model based controller (top) and the model based controller with regulatory layer (bottom).

Figure 2: Controller algorithm, described in terms of the timers which are implemented in Matlab R2013b. The values of the controller parameters Folimit and $\mathrm{Kc}$ are not provided for confidentiality reasons. They were determined based on experience with the process.

Figure 3: Start mass and end mass for experiments 1 and 2 and tanks 1-4. Solid line shows the target mass, and the dashed lines show the $5 \%$ and $10 \%$ deviation from this target mass.

Figure 4: Batch phase mass change $(\mathrm{kg})$ for Experiment 1 (solid lines) and 2 (dashed lines) due to evaporation. In the batch phase of the operation all the tanks have the same operating conditions and no feed is added so mass change is only due to evaporation. The rate of evaporation from Experiment 2 is greater than Experiment 1, due to external air conditions. Time not shown for confidentiality reasons.

Figure 5: Dissolved oxygen profile for experiment 1 and 2. Tanks 3 and 4 have poor oxygen transfer conditions (shown in Table 1), which is shown to cause oxygen limitation in the final hours of the fermentation despite the use of dissolved oxygen control. Time not shown for confidentiality reasons.

Figure 6: End weight of the four experiments conducted. The target fill is shown as a solid red line, and $5 \%$ and $10 \%$ deviations from the target are shown as dashed lines.

Figure 7: Dissolved oxygen (top) and feed rate profiles (bottom) for model based control strategy (experiment 3, left) and the model based control strategy with regulatory layer (experiment 4 , right). The results show that the addition of the regulatory layer successfully adjusts the model-calculated feed rate in order to avoid oxygen limited conditions.

Figure 8: Dissolved oxygen (top) and mass profiles (bottom) for dissolved oxygen control (experiments 1 and 2, left) and the model based control strategy with regulatory layer (experiment 4 , right). The results show that the target mass is reached with low variance in experiment 4 and the dissolved oxygen profiles are also quite reproducible between the tanks. There is a high variance in both the dissolved oxygen profile and the final mass in the dissolved oxygen controlled batches. 
Figure 9: The end product concentration for experiments 1-4 and tanks 1-4 where the results are arranged by the tank number for easier comparison. The standard deviation on the mean for the final product concentrations is $4.1 \%, 7.8 \%, 1.0 \%$ and $5.0 \%$ for tanks $1,2,3$ and 4 respectively. This shows that the product concentration is not significantly affected either positively or negatively by the model based control strategy. 
Model-based control

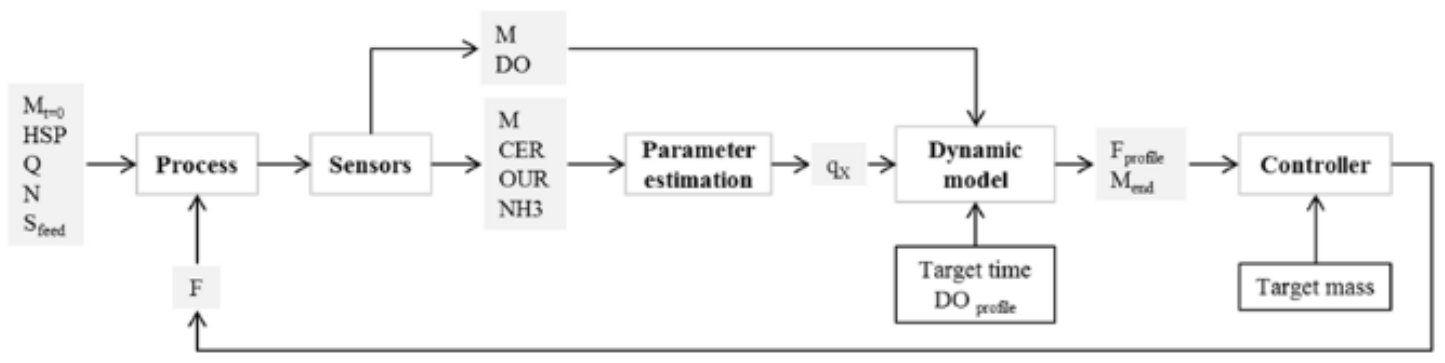

Model-based control with regulatory layer

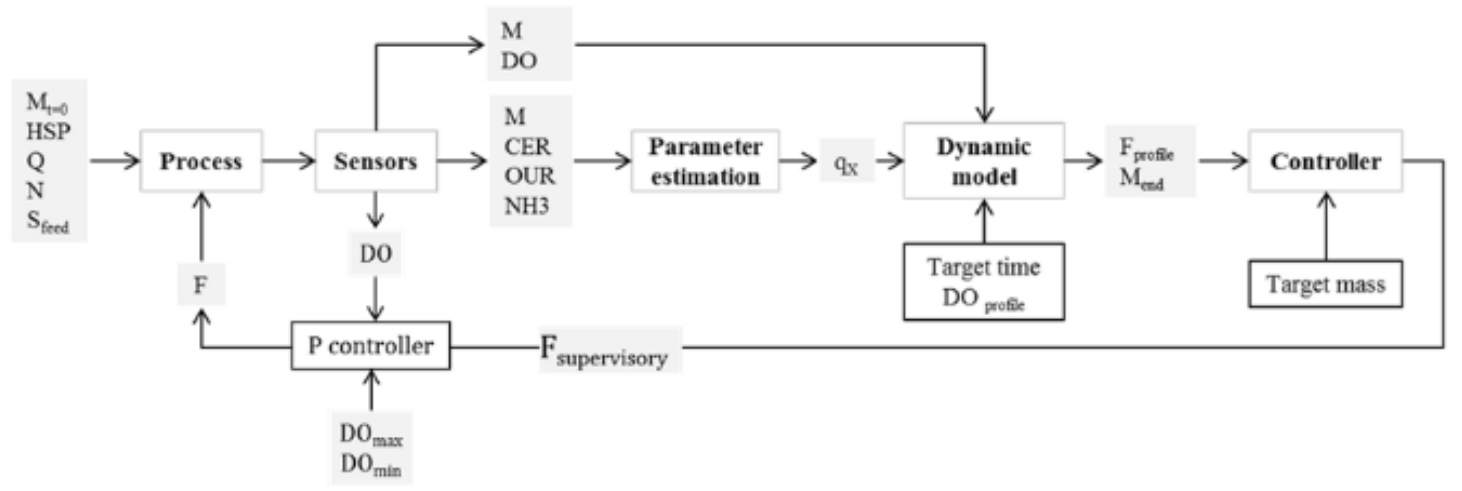

Figure 1 


\section{Start controller:}

- Establish OPC server connection

Initialise MATLAB $B$ timer objects

L Load batch conditions $\left(\mathrm{HSP}_{\max }, \mathrm{Q}_{\max }, \mathrm{N}_{\max }\right)$

L Load model parameters

- Pre-allocate size of array for data storage

Define $\mathrm{DO}_{\text {set }}=\mathrm{f}(\mathrm{t}), \mathrm{DOmin} \mathrm{set}_{\text {set }}=\mathrm{f}(\mathrm{t}), \mathrm{DOmax} \mathrm{set}_{\mathrm{se}}=\mathrm{f}(\mathrm{t}), \mathrm{t}_{\text {end }}$ and $\mathrm{M}_{\text {target }}$

\section{口 Start timers}

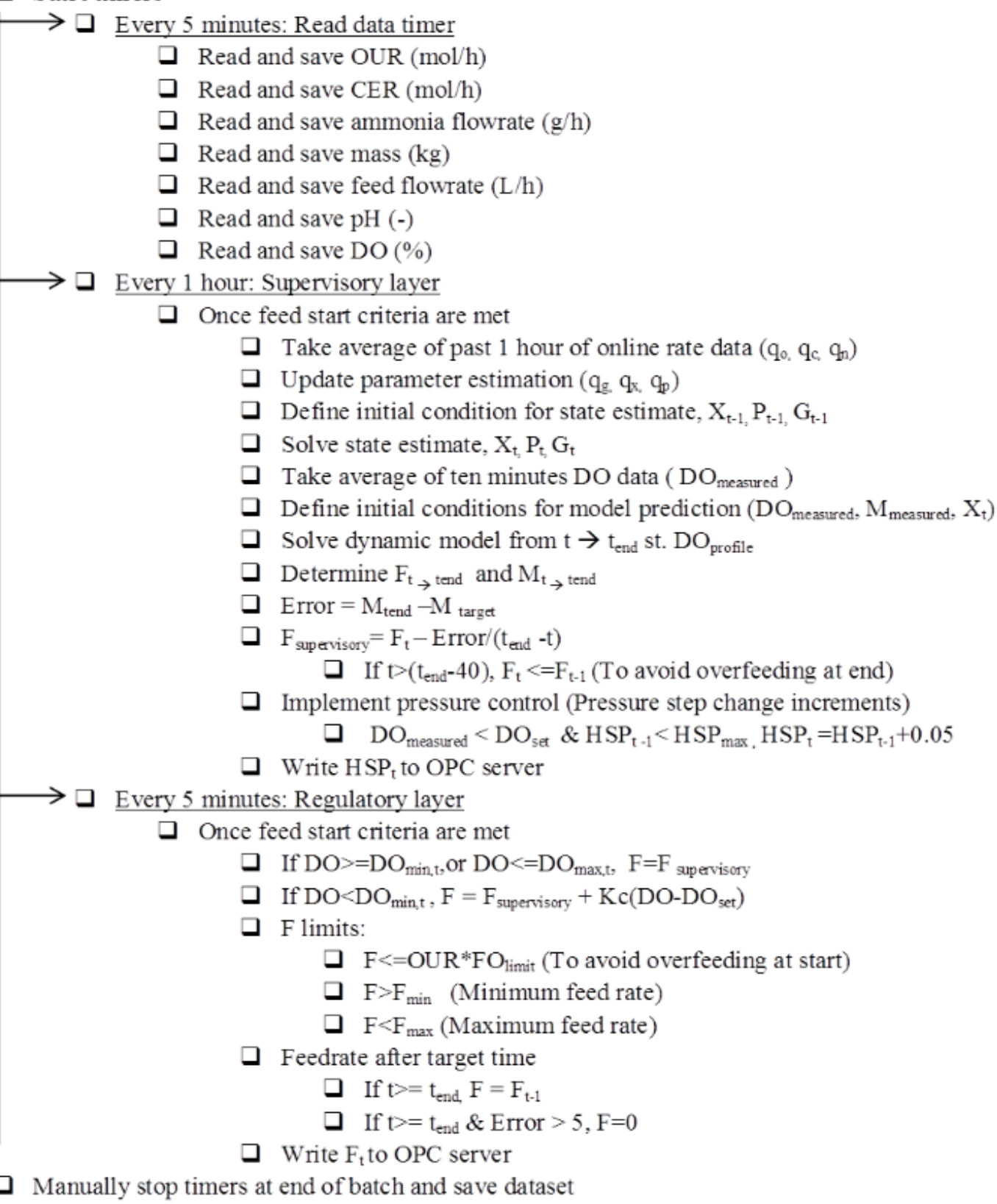

\section{Figure 2}



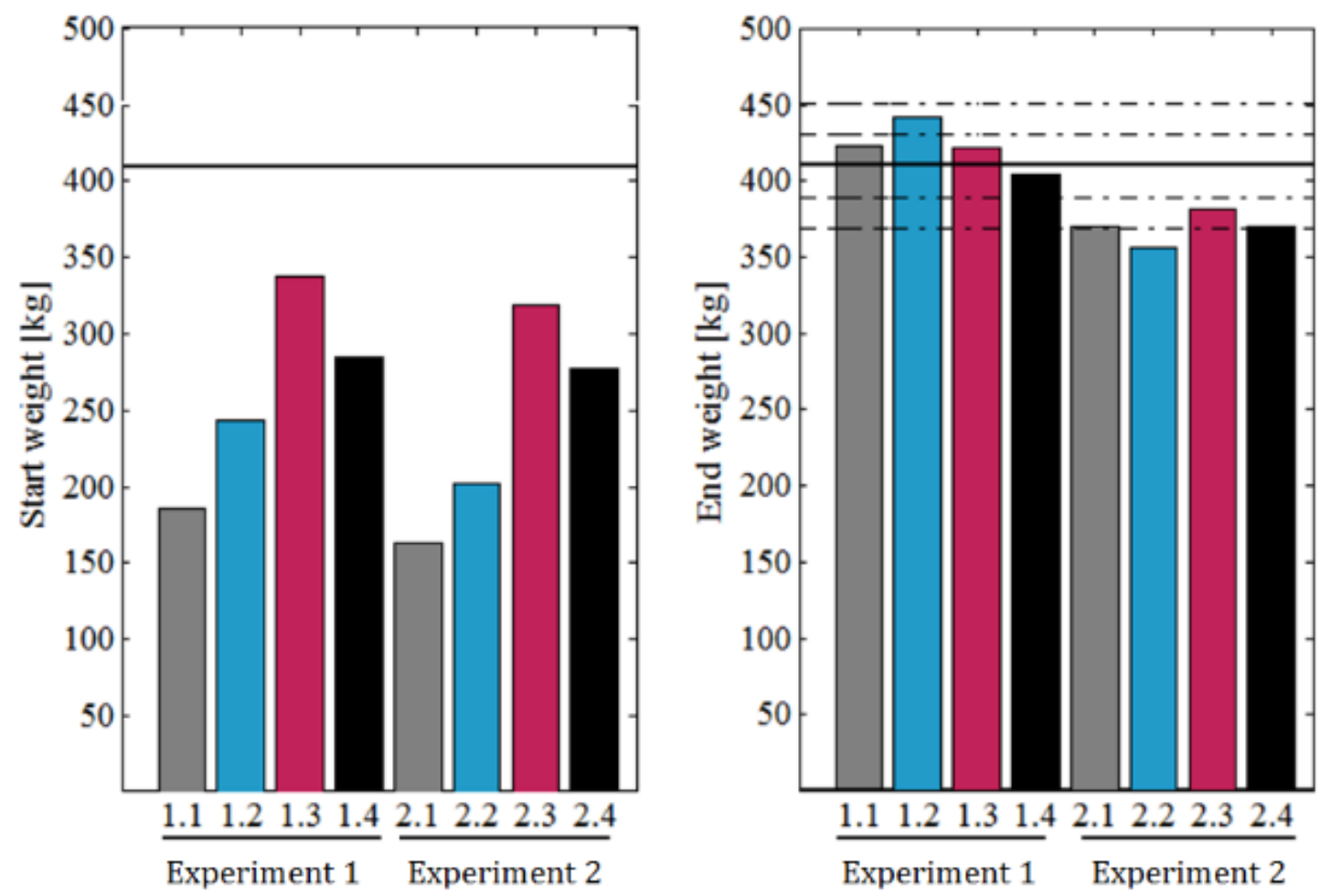

Figure 3 


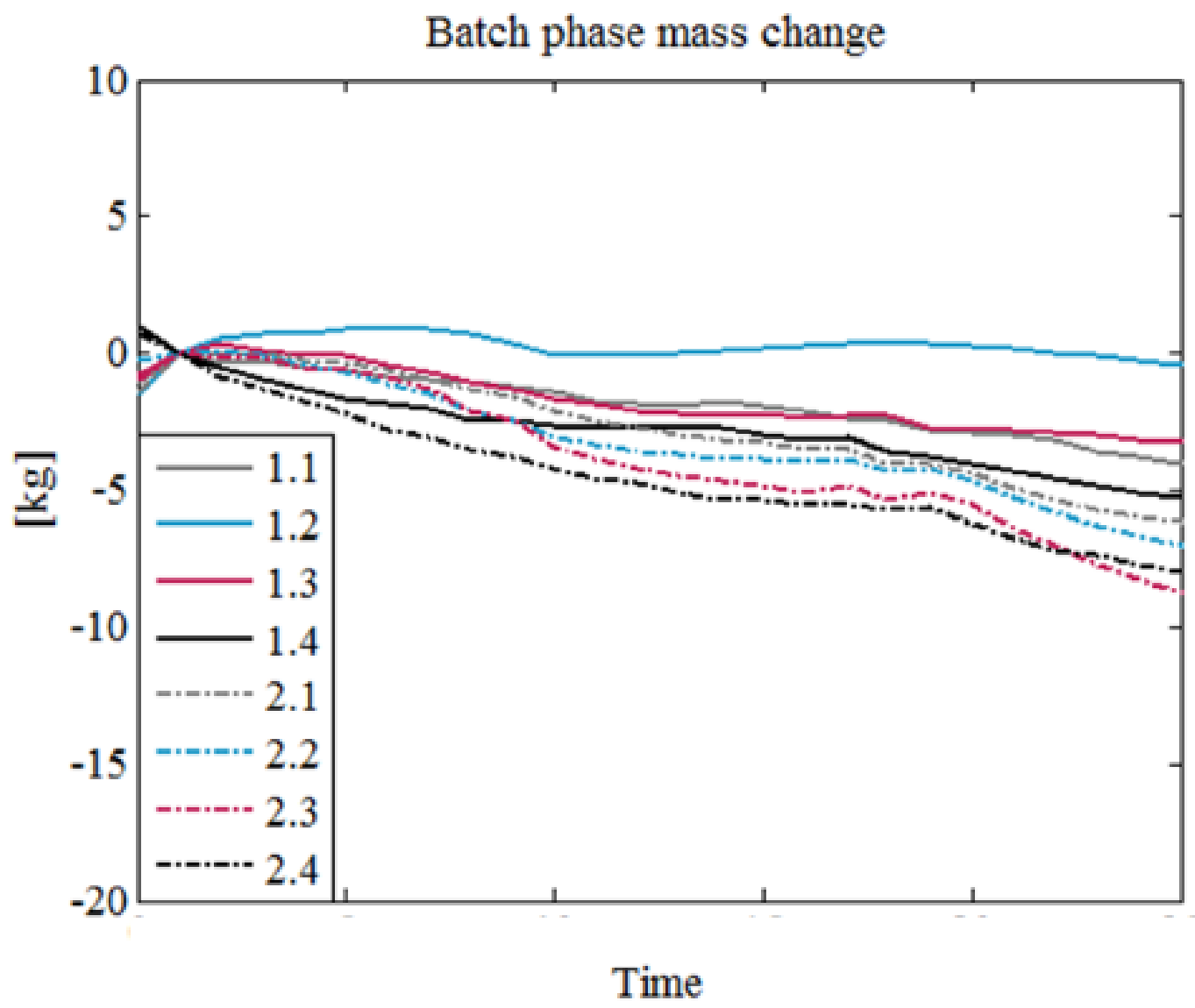

Figure 4 


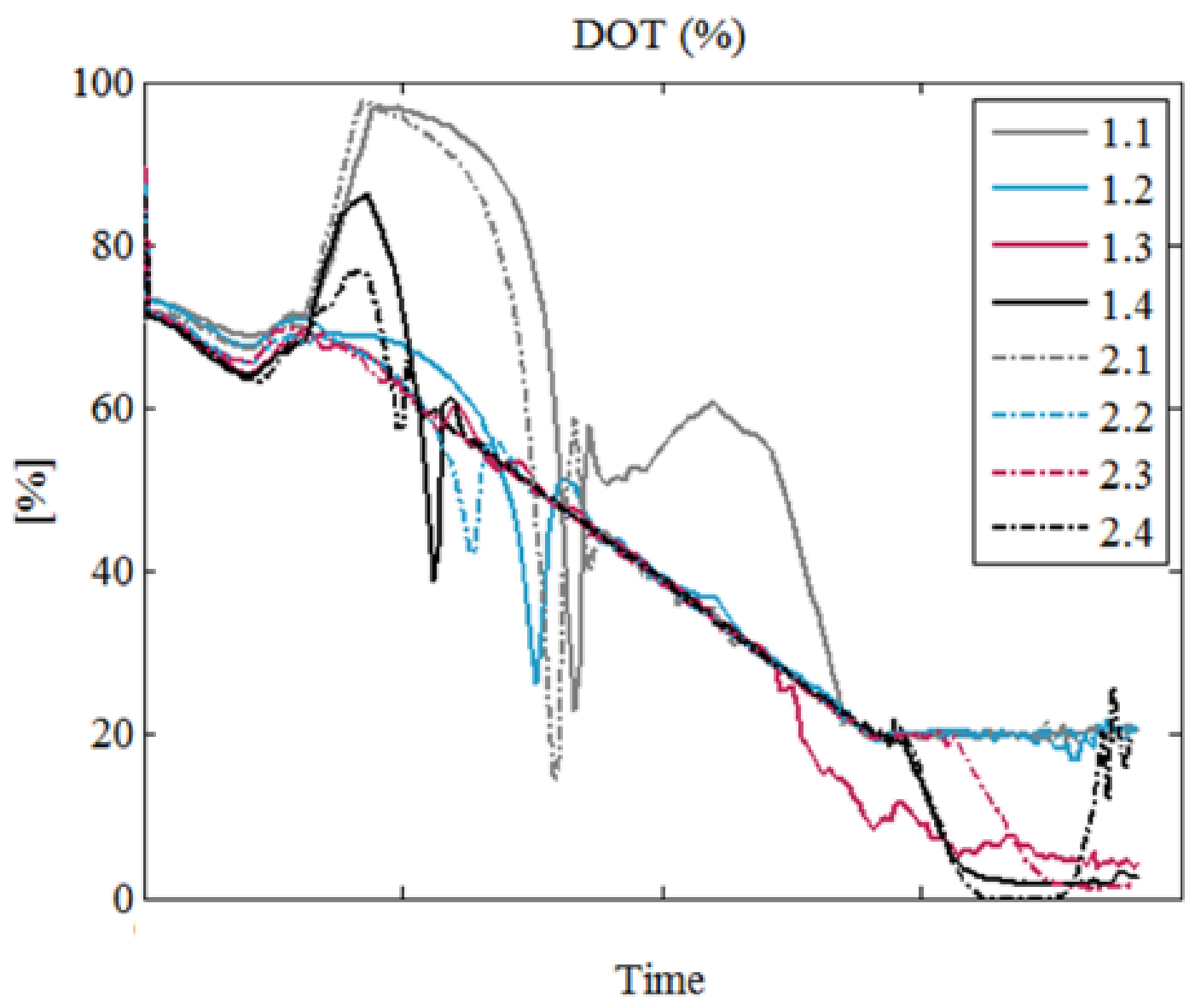

Figure 5 


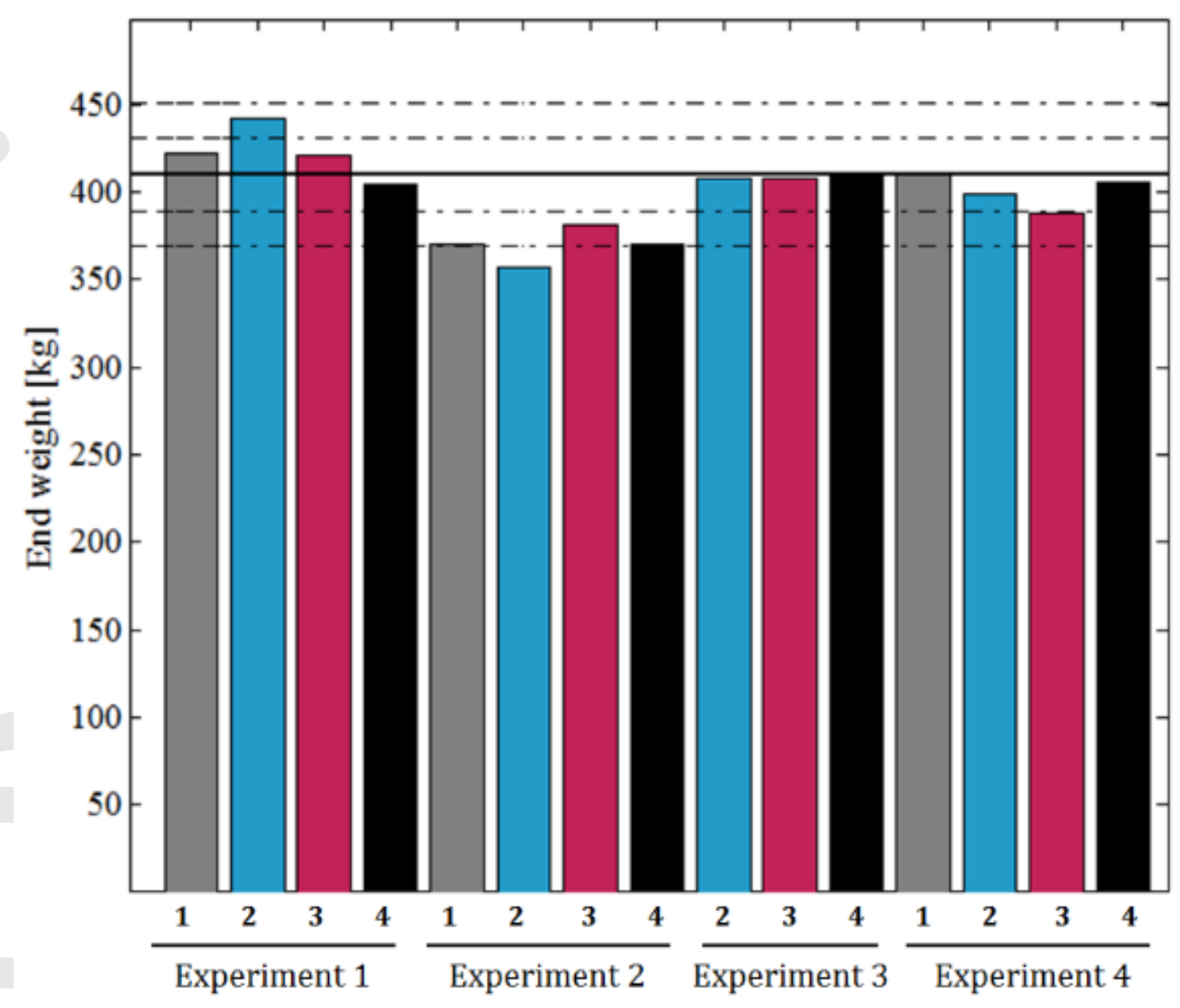

Figure 6 
Experiment 3:

Model based control
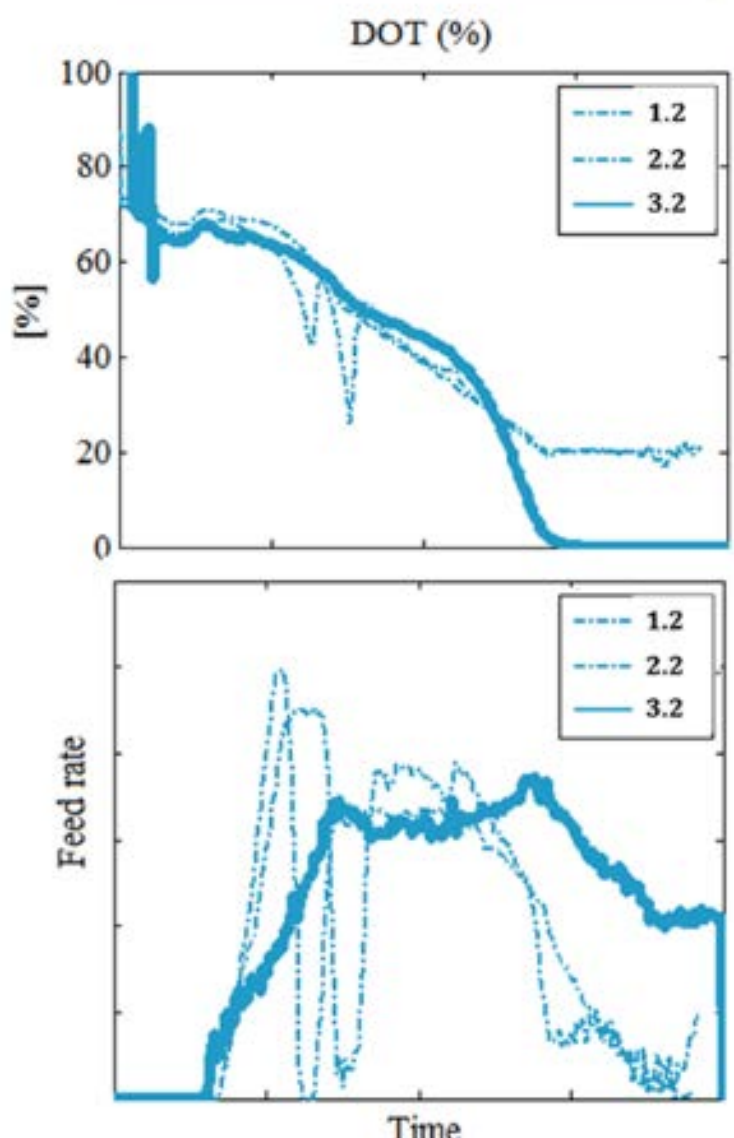

Time
Experiment 4:

Model based control

with regulatory layer
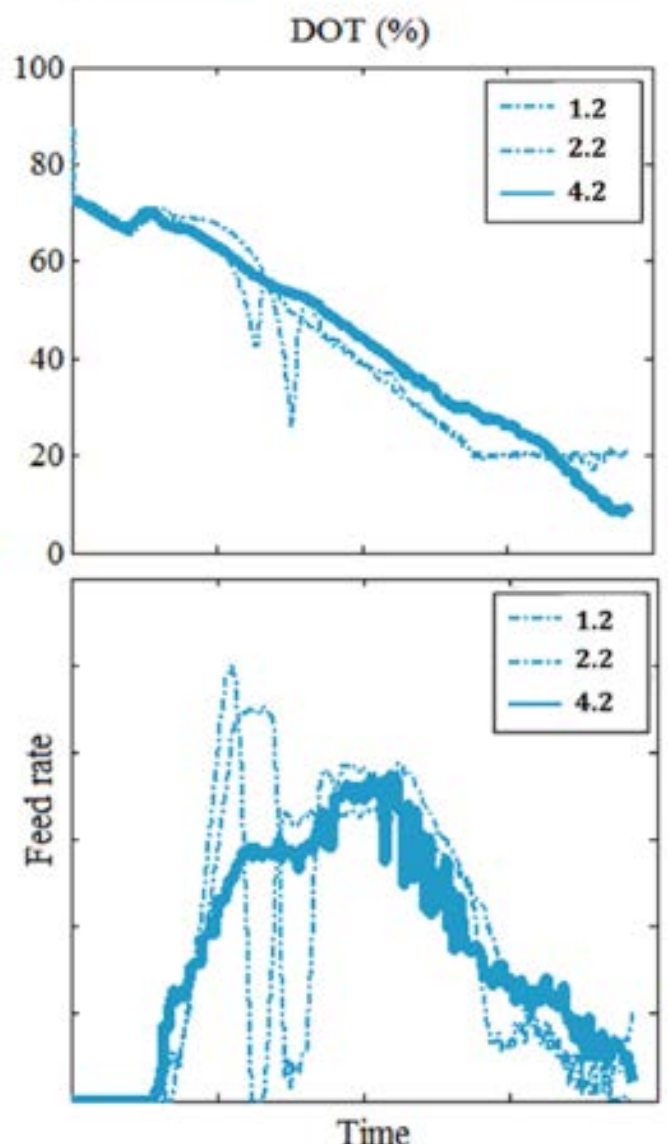

Figure 7 
Experiments 1 \& 2: Do control
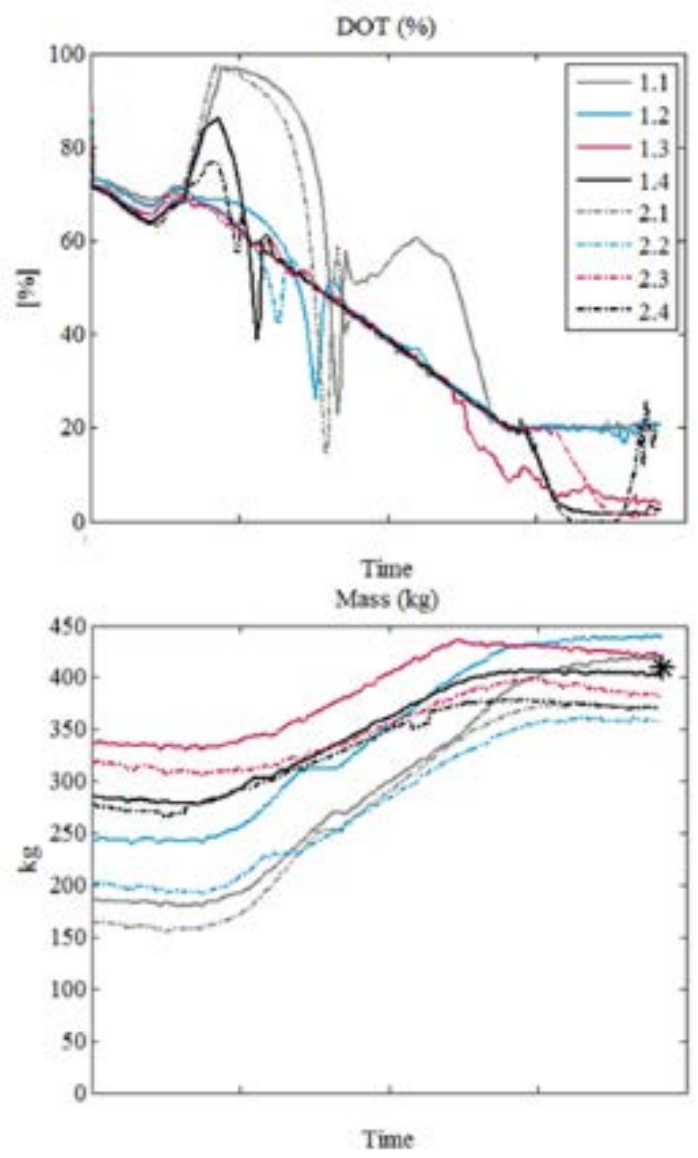

Experiment 4:

Model-based control

with regulatory layer
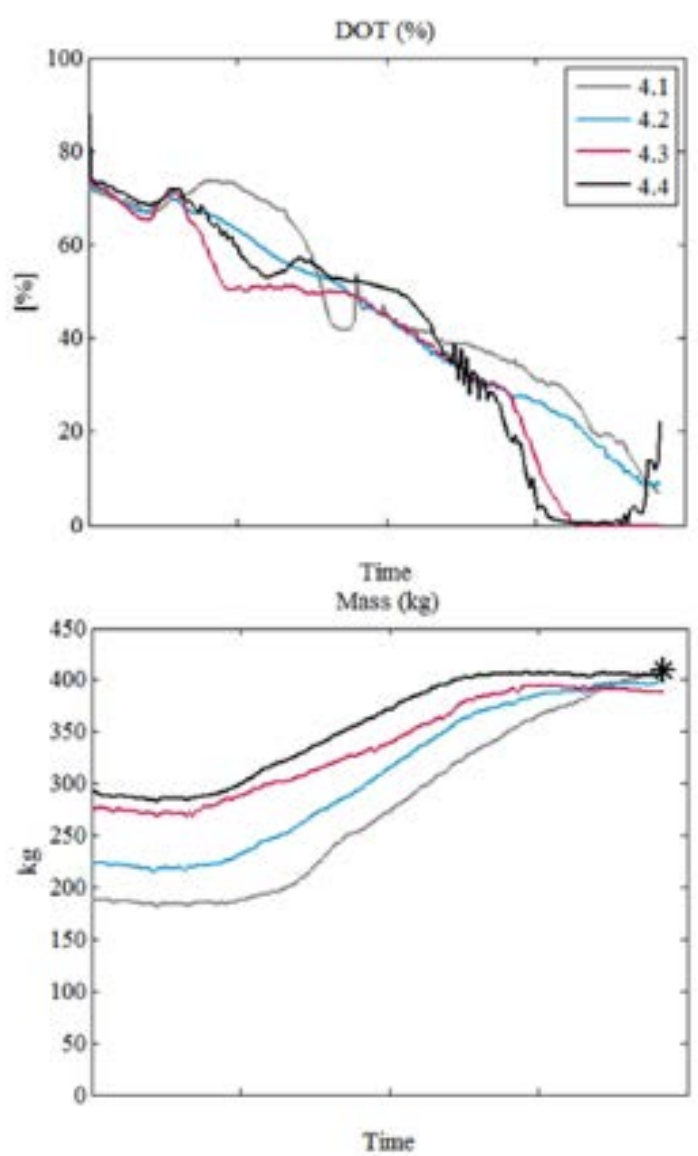

Figure 8 


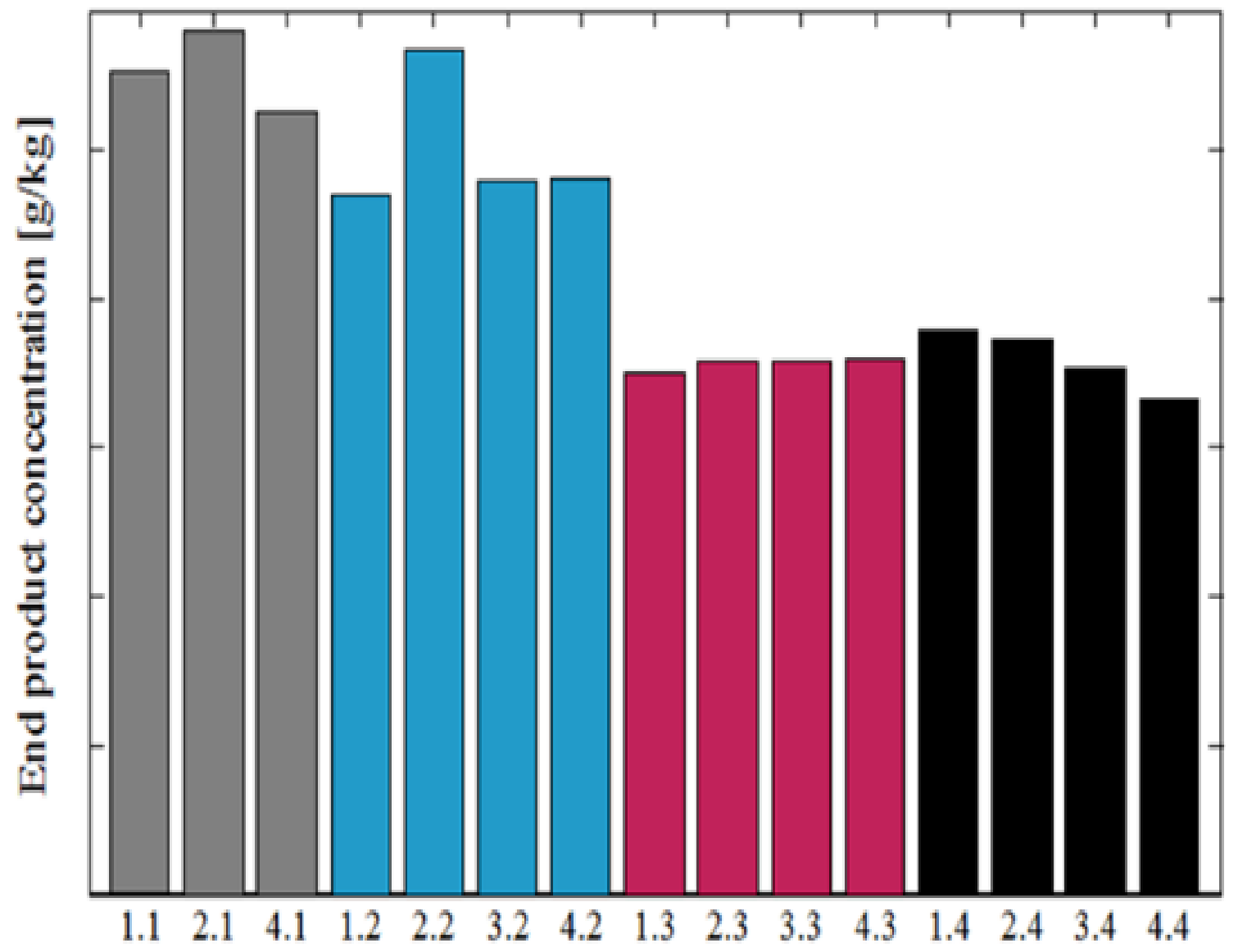

Figure 9 


\begin{tabular}{cccccc} 
Plot colour & Tank & Stirrer speed & Aeration rate & Pressure & Start fill \\
\hline & 1 & + & + & + & $39 \%$ \\
2 & + & - & - & $49 \%$ \\
3 & - & + & - & $77 \%$ \\
4 & - & - & + & $67 \%$ \\
\hline
\end{tabular}

Table 1: Start fill calculation using mechanistic process model for different processing conditions.

Process conditions are not shown for confidentiality reasons. Start fill is relative to the target mass. 